Review

\title{
Wide and Fast Wavelength-Swept Fiber Lasers Based on Dispersion Tuning and Their Application to Optical Coherence Tomography
}

\author{
Shinji YAMASHITA* and Yuy a TAKUBO \\ Research Center for Advanced Science and Technology (RCAST), The University of Tokyo, 4-6-1 Komaba, Meguro-ku, \\ Tokyo 153-8904, Japan \\ "Corresponding author: Shinji YAMASHITAＥ-mail: syama@ee.t.u-tokyo.ac.jp
}

\begin{abstract}
We recently proposed a new class of wavelength-swept lasers for swept-source optical coherence tomography (SS-OCT). It uses the same gain medium with the conventional swept lasers, but does not require any tunable filters. It is based on a principle called "dispersion tuning", in which a highly dispersive medium is inserted in the laser cavity, and loss/gain modulation is applied to mode-lock the laser. Since the cavity contains no mechanical components, such as tunable filters, we could achieve the very high sweep rate. In this review paper, we describe the principle of the dispersion-tuned swept lasers in detail and present our recent work on the application to the SS-OCT system.
\end{abstract}

Keywords: OCT, swept lasers, dispersion tuning

Citation: Shinji YAMASHITA and Yuya TAKUBO, "Wide and Fast Wavelength-Swept Fiber Lasers Based on Dispersion Tuning and Their Application to Optical Coherence Tomography," Photonic Sensors, DOI: 10.1007/s13320-013-0129-0.

\section{Introduction}

Optical coherence tomography (OCT) is a noninvasive cross-sectional imaging technique using infrared light, which can be used to obtain images of the tissue with the micron scale resolution [1]. Especially, the swept-source OCT (SS-OCT) using a wavelength-swept laser has attracted great attention in recent years due to its large imaging depth and high imaging speed $[2,3]$. In the SS-OCT system, the scattered and reflected light from the object under test, typically a living tissue, is resolved in the frequency domain by interference with the reference light, as shown in Fig. 1.

The spatial resolution is inversely proportional to the sweep wavelength range, and the imaging speed is determined by the sweep rate of the swept source. Thus, the performance of the swept source determines the system performance. For the real-time three-dimensional (3D) OCT measurement, the wide tuning range over $100 \mathrm{~nm}$ and fast sweep rate over $100 \mathrm{kHz}$ are typically required.

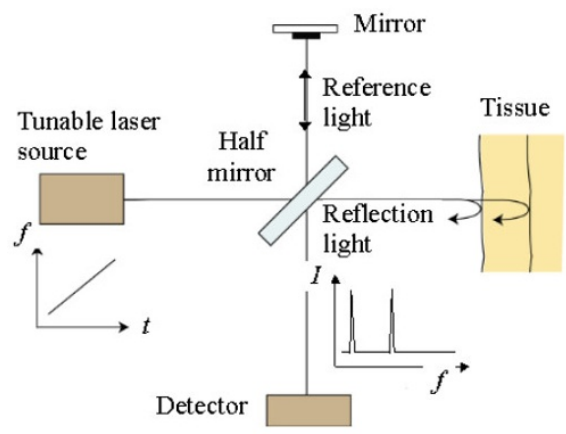

Fig. 1 Swept-source optical coherence tomography.

Received: 16 July 2013 / Revised version: 29 August 2013

C The Author(s) 2013. This article is published with open access at Springerlink.com 
Up to now, a number of broadband swept light sources for the SS-OCT have been reported [4-9]. The most widely used wavelength-swept lasers consist of a wide gain medium and a fast tunable optical filter, as illustrated in Fig. 2(a). The wide gain medium is typically a semiconductor optical amplifier (SOA), which can work at many wavelength bands $(1550 \mathrm{~nm}, 1300 \mathrm{~nm}, 1000 \mathrm{~nm}$, and $800 \mathrm{~nm}$ ) with the wide gain bandwidth over $100 \mathrm{~nm}$. As for the sweep rate, there are two limiting factors, one is the sweep speed of the optical filters, and the other is the cavity length (typically a few tens of meters). There have been several fast tunable optical filters, such as rotating polygonal mirror scanners $[4$, 5], or tunable Fabry-Perot filters (FFPs) based on the piezoelectric actuator (PZT) or micro-electromechanical system (MEMS) [6, 7]. They are basically mechanically tunable filters, in which the sweep speed is normally limited below a few tens of $\mathrm{kHz}$ by the mechanical movements. FFPs have been shown to be able to be swept much faster, a few hundreds of $\mathrm{kHz}$ [8-11], but the durability might be a problem, and the linearity of the sweep in the frequency domain is not warranted. The second limiting factor, the cavity length, has two solutions. One is to make the cavity as short as possible. An ultimate example is the single-mode MEMS-tunable vertical-cavity surface emitting laser (VCSEL) [12] where the cavity length is equal to the FFP length. The other solution is the use of the so-called Fourier-domain mode locking (FDML), where the cavity length is elongated (typically a few kilometers) so that the sweep time is set to be equal to one round trip time of the cavity [8]. Using this method, the sweep rate has been improved significantly [8-11].

We proposed another class of wavelength-swept lasers based on a principle called "dispersion tuning" [13]. It uses the same gain medium with the conventional swept lasers, but does not require any tunable filters. Instead, we insert a highly dispersive medium in the laser cavity and apply loss/gain modulation to mode-lock the laser, as illustrated in Fig. 2(b). Since the cavity contains no mechanical components, such as tunable filters, we could achieve very high sweep rate. We have applied the swept laser for the dynamic fiber Bragg grating (FBG) sensors $[14,15]$. In this review paper, we describe the principle of the dispersion-tuned swept lasers in detail and present our recent work on application to the SS-OCT system $[16,17]$.

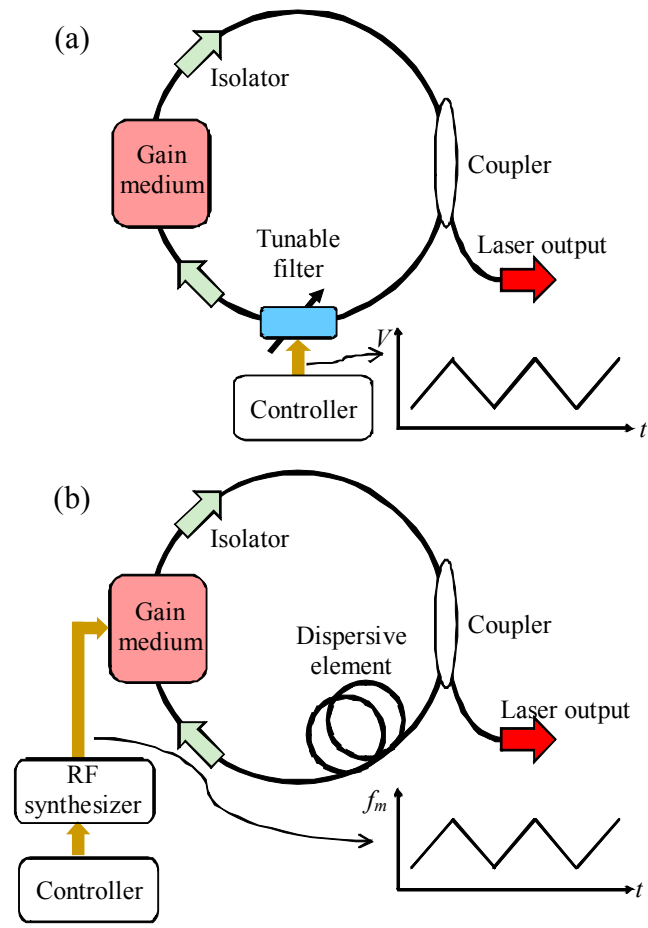

Fig. 2 Wavelength-swept fiber lasers: (a) conventional laser and (b) dispersion-tuned laser.

\section{Principle}

\subsection{Dispersion tuning}

The laser cavity has discrete longitudinal resonance modes. The resonance condition of the ring cavity is expressed as

$$
\beta\left(\omega_{K}\right) L=2 K \pi
$$

where $K$ is the mode number, $\beta(\omega)$ denotes the propagation constant at an angular frequency $\omega, \omega_{K}$ is the angular frequency of the $K$-th resonance mode, and $L$ is the cavity length. Equation (1) can be transformed to

$$
\left[\beta\left(\omega_{K+1}\right)-\beta\left(\omega_{K}\right)\right] L=2 \pi
$$


The spacing between adjacent modes or the free-spectral range (FSR) $F$ of the laser cavity is expressed, using the relation $\beta=n \omega / c$, as

$$
F=\frac{\omega_{K+1}-\omega_{K}}{2 \pi}=\frac{c}{n L}
$$

where $n$ is the effective refractive index in the cavity, and $c$ is the speed of light in the vacuum. Note that $n$ is assumed to be a constant in deriving (3).

In the fiber laser cavity, $L$ is long, thus the spacing between adjacent modes is small. Here, we assume $\omega_{K+1}-\omega_{K}<<\omega_{K}$, then, (2) becomes

$$
\left(\omega_{K+1}-\omega_{K}\right) \frac{d \beta}{d \omega} L=2 \pi .
$$

Equation (4) leads to another but more general expression of the FSR, as

$$
F=\left[\frac{d \beta}{d \omega} L\right]^{-1} .
$$

We can lock the lasing modes and generate a short pulse train by applying the modulation at a frequency, which is well known as the active mode locking technique [18]. The modulation frequency $f_{m}$ applied to the cavity has to match with an integer $(N)$ times of the FSR $(=N \times F)$, where $N$ is the order of harmonics (harmonic mode locking). Without the chromatic dispersion in the cavity, $F$ is a constant over entire frequency. In this case, the lasing occurs at the gain peak wavelength, regardless of whether the modes are locked or not.

When the chromatic dispersion exists in the laser cavity, $F$ becomes dependent on the frequency, $F(\omega)$. By expanding $\beta(\omega)$ around a frequency $\omega_{0}$ with the Taylor series, (5) becomes

$$
\begin{aligned}
F(\omega) & =\frac{1}{L}\left[\beta_{1}+\beta_{2}\left(\omega-\omega_{0}\right)+\frac{1}{2} \beta_{3}\left(\omega-\omega_{0}\right)^{2}+\cdots\right]^{-1} \\
& \approx F_{0}\left[1-\frac{\beta_{2}}{\beta_{1}} \Delta \omega-\frac{1}{2} \frac{\beta_{3}}{\beta_{1}} \Delta \omega^{2}-\cdots\right]
\end{aligned}
$$

where $F_{0}=1 /\left(\beta_{1} L\right)$, which is the FSR at $\omega=\omega_{0}$, and $\Delta \omega=\omega-\omega_{0}$. Here, we assume that the terms containing $\Delta \omega$ are much smaller than the first term. Neglecting the higher order chromatic dispersion $\left(\beta_{3}\right.$, $\left.\beta_{4}, \cdots\right)$, the change in the FSR from $F_{0}$ by the change in the frequency, $\Delta F(\omega)$, is expressed as

$$
\Delta F(\omega)=-F_{0} \frac{\beta_{2}}{\beta_{1}} \Delta \omega .
$$

Equation (7) implies that the FSR is enlarged or reduced (depending on the sign of $\beta_{2}$ ) linearly in proportion to the change in the frequency, as shown in Fig. 3. The dispersion tuning is a technique using the unevenly spaced resonance modes in the dispersive laser cavity $[19,20]$. Supposing that we apply a modulation at $f_{m}$ to the dispersive cavity strongly enough to be mode locked, the laser is obliged to operate at a frequency to meet the mode-locking condition. In the case of Fig. 3 where $\beta_{2}$ is negative, the lower $f_{m}$ stimulates lasing at the lower frequency, and the higher $f_{m}$ stimulates lasing at the higher frequency. Reminding (7) and the harmonic mode locking of the $N$-th order, the change in the lasing frequency $\Delta \omega$ and the change in the modulation frequency $\Delta f_{m}$ should have a relation:

$$
\Delta \omega=-\frac{\beta_{1}}{N \beta_{2} F_{0}} \Delta f_{m}=-\frac{n_{0}}{c \beta_{2} f_{m 0}} \Delta f_{m}
$$

where $f_{m 0}=N F_{0}$, which is the initial modulation frequency to give $\omega=\omega_{0}$. Here, we use $\beta_{1}=n_{0} / c$, and $n_{0}$ is the refractive index at $\omega=\omega_{0}$. From (8), it is found that the lasing frequency can be swept linearly by sweeping the modulation frequency.

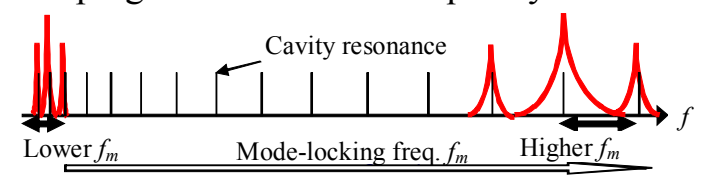

Fig. 3 Principle of dispersion tuning: unevenly spaced resonance modes in the dispersive laser cavity.

It is sometimes more useful to rewrite (8) in terms of the change in the lasing wavelength $\Delta \lambda$. Using relations $\Delta \omega=-2 \pi c \Delta \lambda / \lambda^{2}, \quad \beta_{2}=$ $-\lambda^{2} D / 2 \pi c$, where $D$ is the dispersion parameter (in $\mathrm{ps} / \mathrm{nm} / \mathrm{km}$ ), (8) can be transformed to

$$
\Delta \lambda=-\frac{n_{0}}{c D f_{m 0}} \Delta f_{m}=-\frac{n_{0} L}{c D_{\text {total }} f_{m 0}} \Delta f_{m}
$$

where $D_{\text {total }}=D L$ is the total amount of intracavity dispersion (in $\mathrm{ps} / \mathrm{nm}$ ). Thus, the lasing frequency or wavelength can be tuned by changing the modulation frequency. This is called the dispersion tuning technique $[19,20]$. It is found from (9) that 
the wavelength shift is more sensitive to the change in the modulation frequency when $D$ and $f_{m 0}$ are small, which means that the wavelength is not well defined, and dispersion tuning is weak.

The wavelength sweep range $\Delta \lambda_{\max }$ is determined by two factors. One is the gain bandwidth, and the other is lasing at the adjacent harmonic mode, $(N-1)$-th or $(N+1)$-th mode. It happens when the change in the modulation exceeds one FSR. From (9), $\Delta \lambda_{\max }$ is expressed as

$$
\Delta \lambda_{\max }=\frac{1}{|D| L f_{m 0}}=\frac{1}{\left|D_{\text {total }}\right| f_{m 0}} .
$$

Equation (10) means that the smaller $D, L$, and $f_{m 0}$ give the wider sweep range.

The sweep rate $v_{\mathrm{sw}}$ is inversely proportional to the photon lifetime as is the case of conventional swept lasers, that is, the sweep speed is faster when $L$ is small:

$$
v_{\mathrm{sw}} \propto \frac{1}{L} .
$$

Since the laser is mode locked, the output is not in the continuous wave (CW), but is pulsed at the repetition rate equal to the modulation frequency. The pulse is strongly chirped and wide by the intracavity dispersion. The pulse from the mode-locked fiber laser with the intracavity dispersion is expressed with a chirped Gaussian pulse [20], whose spectral half width $\delta \omega$ is given by

$$
\delta \omega=\left(\pi \frac{f_{m 0}}{\lambda}\right)^{1 / 2}\left(\frac{8 \pi c M}{|D| L}\right)^{1 / 4}
$$

where $M$ is the modulation depth. This equation means that the linewidth can be narrower as $f_{m 0}$ is smaller, and $D$ and $L$ are larger. However, from (9), the smaller $f_{m 0}$ increases the instability of the lasing wavelength and causes linewidth broadening, which is the major effect in the following experiments. Thus, $f_{m 0}$ should be set as high as the linewidth broadening by (12) can be ignored.

From the above discussions, in order to realize wide and fast tuning with the narrow spectral linewidth, $D$ and $f_{m 0}$ should be as large as possible, and $L$ should be as small as possible.

\subsection{OCT performance}

The performance of the OCT system is determined by the resolution, depth range, imaging speed, and sensitivity. For a tuning source with a Gaussian-profile spectral envelope, the axial resolution of an OCT system can be expressed as [21]

$$
\delta z=\frac{2 \ln 2}{\pi} \frac{\lambda_{0}^{2}}{n \Delta \lambda}
$$

where $n$ is the refractive index of the sample, $\lambda_{0}$ is the central wavelength, and $\Delta \lambda$ is the full width at the half maximum (FWHM) of the spectral envelope (tuning range). As (13) indicates, the axial resolution is inversely proportional to the sweep range. Therefore, a wavelength-swept laser with the wide tuning range is needed.

The coherence length of the SS-OCT system is defined as twice the depth at which the signal intensity decreases by $6 \mathrm{~dB}$. The relation between the coherence length $l_{c}$ and the instantaneous linewidth of the laser $\delta \lambda$ can be expressed as [22]

$$
l_{c}=\frac{2 \ln 2}{\pi} \frac{\lambda_{0}^{2}}{\delta \lambda}
$$

which indicates that a narrower instantaneous linewidth is necessary for a deeper imaging range.

The imaging speed of the SS-OCT system is proportional to the sweep rate of the laser. It also depends on the number of pixels needed for images. For applications such as endoscopy and vascular catheters, short inspection time is crucial, therefore, wavelength-swept lasers with the over $100-\mathrm{kHz}$ sweep rate are in strong demand. The sensitivity of the system is the signal-noise ratio of the OCT signal, and a laser with the high power and low noise is needed for the high sensitivity.

\section{Dispersion-tuned swept fiber laser using the DCF}

\subsection{Setup}

Firstly, we chose to use the dispersion compensating fiber (DCF) as the intracavity 
dispersive element [16]. Figure 4 shows the setup of the dispersion-tuned wavelength swept fiber laser. The semiconductor optical amplifier (SOA) is directly modulated for the mode-locking, and the 100-m-length DCF is inserted to increase the dispersion of the cavity. The dispersion parameter $D$ is about $-120 \mathrm{ps} / \mathrm{nm} / \mathrm{km}$, and the central modulation frequency $f_{m 0}$ is $461 \mathrm{MHz}$. The cavity includes the polarization controller (P.C.) and isolator, and half of the light in the cavity is output via 50:50 couplers. In order to sweep the lasing wavelength, the modulation frequency $f_{m}$ is linearly swept around the central frequency by the function generator (FG).

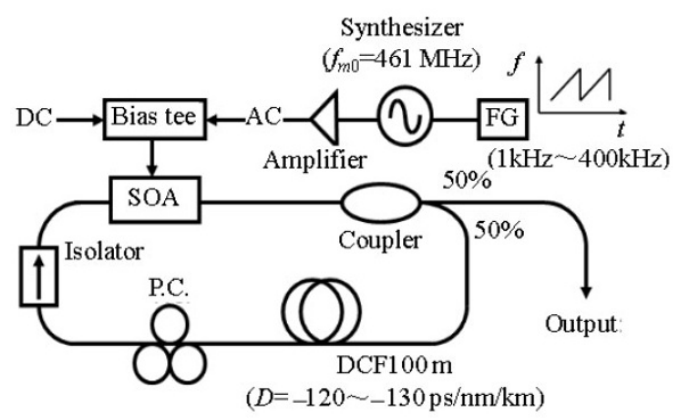

Fig. 4 Dispersion-tuned swept fiber laser using the DCF.

\subsection{Laser characteristics}

In consideration of the radio-frequency (RF) modulation characteristics of the adopted SOA, we set the modulation frequency at about $460 \mathrm{MHz}$. The SOA drive current is $80 \mathrm{~mA}$, and the $\mathrm{AC}$ modulation signal from the synthesizer is $28 \mathrm{dBm}$ after amplification. Figure 5 shows the static characteristics of the dispersion-tuned wavelengthswept fiber laser. Figure 5(a) shows the lasing spectra. As the mode-locking frequency is adjusted manually, the spectra change a wide range of $140 \mathrm{~nm}$. Figure 5(b) shows the relation between the lasing wavelength and the mode-locking (ML) frequency. As the figure indicates, the lasing wavelength is proportional to the mode-locking frequency. Therefore, the triangular waveform from the FG enables the linear sweep of the laser. The output power at $1300 \mathrm{~nm}$ is $7.96 \mathrm{~mW}$, according to the optical power meter. The linewidth at $1300 \mathrm{~nm}$ is $0.61 \mathrm{~nm}$.

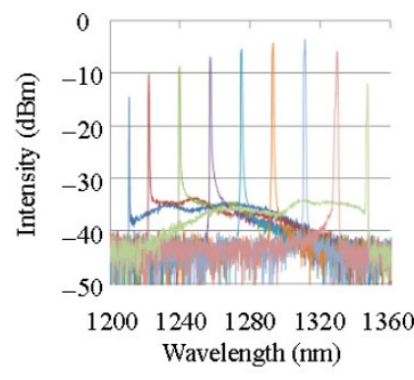

(a)

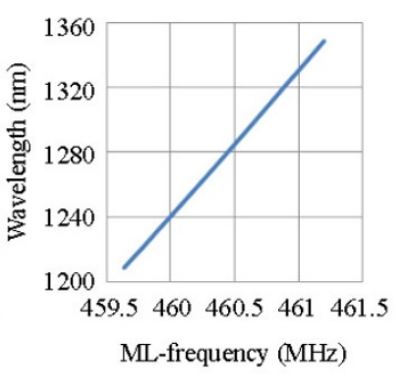

(b)
Fig. 5 Static characteristics: (a) optical spectra and (b) lasing wavelength as a function of the mode-locking frequency.

Figure 6 shows the sweep characteristics obtained by inputting the triangular wave from the function generator and sweeping linearly the mode-locking frequency. Figure 4(a) shows the peak-hold spectra at the sweep rate of $500 \mathrm{~Hz}, 1 \mathrm{kHz}$, $10 \mathrm{kHz}, 100 \mathrm{kHz}, 200 \mathrm{kHz}$, and $400 \mathrm{kHz}$. A decrease in the intensity of the optical spectra is because of the integral time of the peak-hold function of the optical spectrum analyzer. Figure 4(b) shows the temporal waveforms at $1 \mathrm{kHz}, 10 \mathrm{kHz}, 100 \mathrm{kHz}, 200$ $\mathrm{kHz}$, and $400 \mathrm{kHz}$. The output power slightly decreases at the higher sweep rate.

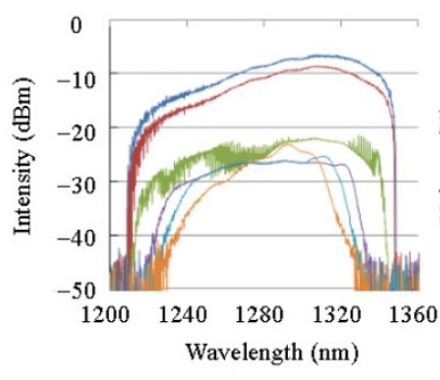

(a)

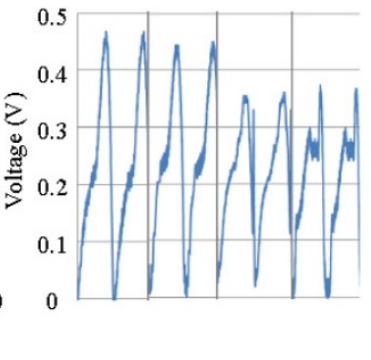

(b)
Fig. 6 Sweep characteristics: (a) peak-hold spectra (from top to bottom: $500 \mathrm{~Hz}, 1 \mathrm{kHz}, 10 \mathrm{kHz}, 100 \mathrm{kHz}, 200 \mathrm{kHz}$, and $400 \mathrm{kHz}$ ) and (b) temporal waveforms of two periods (from left, at $1 \mathrm{kHz}, 10 \mathrm{kHz}, 100 \mathrm{kHz}, 200 \mathrm{kHz}$, and $400 \mathrm{kHz}$ ).

\subsection{Application to SS-OCT}

We applied the dispersion-tuned swept fiber laser to the OCT system. Figure 7 shows the setup of the SS-OCT system. 


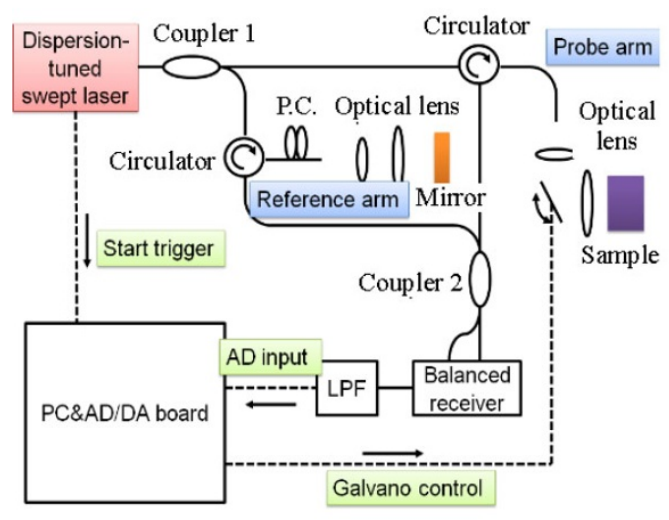

Fig. 7 OCT setup.

Figure 8 shows the point spread function of the laser at the sweep rate of $1 \mathrm{kHz}$. The function is achieved by the following method: inputting the light to the interferometer, one of the split light reflects at the reference mirror, and the other reflects at the $-35-\mathrm{dB}$ anti-reflection(AR)-coated mirror which is set at the sample side of the interferometer. The intensity of the interfering light decreases as the optical path is longer, which corresponds to the imaging depth. The signal near $0 \mathrm{~mm}$ is the system noise.

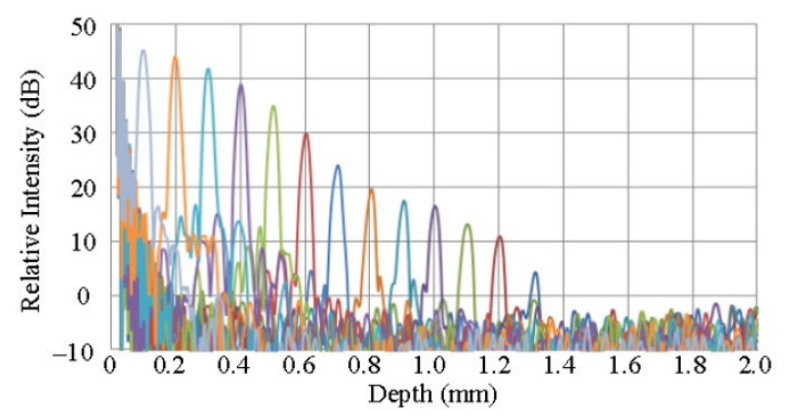

Fig. 8 Point spread function of the dispersion-tuned laser (at the sweep rate of $1 \mathrm{kHz}$ ).

As we showed in the previous chapter, the sweep range $\Delta \lambda$ is $140 \mathrm{~nm}$, and the central wavelength $\lambda_{0}$ is about $1280 \mathrm{~nm}$. Substituting these source parameters into (13), the theoretical axial resolution is calculated to be $5.15 \mu \mathrm{m}$. The axial resolution of the system, which is read from the FWHM of the peak, is $16.0 \mu \mathrm{m}$, which is about three times larger than the theoretical value. The $-6 \mathrm{~dB}$ drop-off depth is $0.38 \mathrm{~mm}$. The sensitivity read out from the graph is $52 \mathrm{~dB}$. As we used the $-35-\mathrm{dB}$ AR-coat mirror, the effective sensitivity is about $87 \mathrm{~dB}$.

Figure 9 shows the OCT image of the human finger. The picture is achieved at the sweep rate of $1 \mathrm{kHz}$. The stripe pattern near the surface derives from the system noise. The sweat gland under the surface of the finger can be observed near the center of the image.

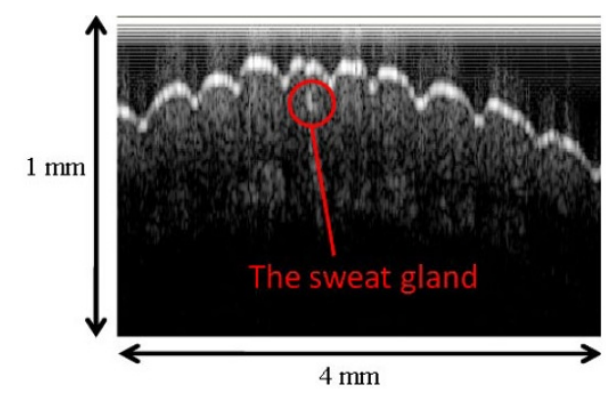

Fig. 9 OCT image of the human finger.

\section{Dispersion-tuned swept fiber laser using CFBG}

\subsection{Setup}

In the previous session, we demonstrated the dispersion-tuned fiber laser at $1.3-\mu \mathrm{m}$ bands and applied it to the SS-OCT system. OCT images of the human finger were successfully obtained at the $1-\mathrm{kHz}$ sweep rate. However, we could not obtain OCT images at higher sweep rates because of the performance degradation of the laser. Since we used 100-m-length DCF as the dispersive element, the total length of the cavity exceeded $100 \mathrm{~m}$. The long cavity resulted in a decrease in the output power and the linewidth broadening at higher sweep rates. In order to enhance the laser performance at higher sweep rates, the cavity length has to be shortened.

In order to shorten the cavity length, we chose to use a chirped fiber Bragg grating (CFBG) and a reflective semiconductor optical amplifier (RSOA) [17]. The dispersion-tuned laser using the CFBG as the dispersive element was first demonstrated by Burgoyne et al. [23-25]. The RSOA has been applied to the dispersion-tuned fiber laser to enhance the modulation and dispersion efficiency [26]. We combined the CFBG and the RSOA to make a linear 
cavity instead of a ring cavity whose length tends to be longer.

Here, we compare the performance of three types of dispersion-tuned fiber lasers shown in Fig. 10. The first setup using the DCF is shown in Fig. 10(a). The laser consists of a ring resonator, including a SOA module, a 100-m-length DCF $(-90 \mathrm{ps} / \mathrm{nm} / \mathrm{km}$ at $1.5-\mu \mathrm{m}$ bands), a P.C., and an isolator. The $10 \%$ of the intracavity light is output via a fiber coupler. The laser output is amplified by the second SOA. The cavity length is $105.2 \mathrm{~m}$. The second configuration shown in Fig. 10(b) is the same setup as the first one except that the CFBG $(-10 \mathrm{ps} / \mathrm{nm}$ at $1.5-\mu \mathrm{m}$ bands) is used with a circulator instead of the DCF. The CFBG is made in a polarization maintaining (PM) fiber and with a reflectivity of $65 \%$. The cavity length is $9.8 \mathrm{~m}$. By combining the RSOA and the CFBG, we can adopt a linear resonator configuration, as shown in Fig. 10(c). The light from the RSOA enters the CFBG, and $65 \%$ of the light is reflected. The remaining $35 \%$ of the light passes through the CFBG and is amplified by the SOA. Since all the fibers used in this configuration are PM fibers, and a P.C. is not needed in the cavity. The cavity length is $2.05 \mathrm{~m}$. Since this is a linear laser, the effective length is doubled to be $4.1 \mathrm{~m}$, less than half of that in the ring laser.

The direct current (DC) for driving the SOA and alternate current (AC) for intensity modulation are injected into the SOA through the bias tee. A synthesizer is used for generating the AC mode-locking signal. As the cavity includes a highly dispersive element, the lasing wavelength changes corresponding to the modulation frequency. This can be confirmed by changing the modulation frequency manually. In order to sweep the laser wavelength, a saw-tooth wave is input to the synthesizer from the FG to sweep the modulation frequency linearly. The output spectra and the temporal waveform were observed by an optical spectrum analyzer and an oscilloscope, respectively.
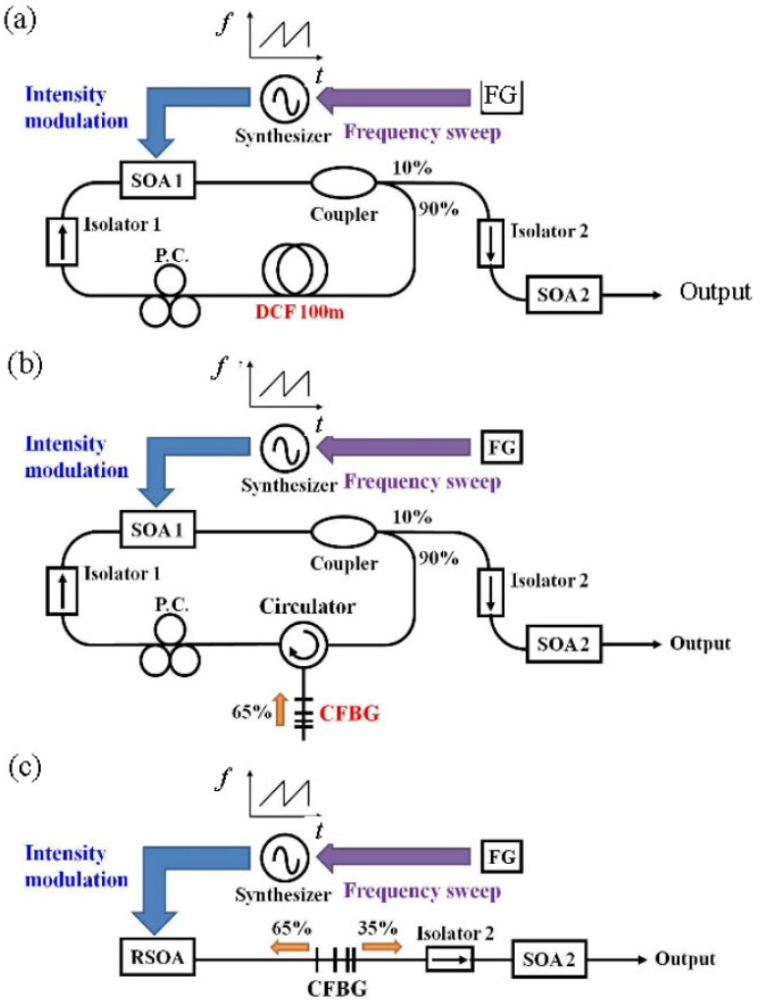

Fig. 10 Setups of dispersion-tuned fiber lasers: (a) setup-A, (b) setup-B, and (c) setup-C.

\subsection{Laser characteristics}

We set the central modulation frequency $f_{m 0}$ at around $411 \mathrm{MHz}, 612 \mathrm{MHz}$, and $770 \mathrm{MHz}$ for the setup-A (SOA+DCF), the setup-B (SOA+CFBG), and the setup-C (RSOA+CFBG), respectively. As we mentioned in Section 2.1, there is a trade off in the choice of $f_{m 0}$ : the lower $f_{m 0}$ leads to the linewidth broadening, and the higher $f_{m 0}$ leads to the limited tuning range. We first measured the responses of the SOA modules as a function of the modulation frequency to find the best $f_{m 0}$. The $f_{m 0}$ chosen in each setup is the highest modulation frequency at which the maximum tuning range is maintained. The drive current to the SOA1 in the setup-A and setup-B is $110 \mathrm{~mA}$, and the drive current to the RSOA in the setup- $\mathrm{C}$ is $55 \mathrm{~mA}$. The drive current to the SOA2 is about $300 \mathrm{~mA}$. The AC modulation signal from the synthesizer is amplified to $28 \mathrm{dBm}$ and input to the SOA via the bias tee.

Figure 11 shows the static tuning characteristics 
of the dispersion-tuned wavelength-swept fiber lasers. Figures 11(a) - 11(c) show the lasing spectra of the setup-A, setup-B, and setup-C observed by the optical spectrum analyzer (OSA). The tuning ranges of each setup are $131 \mathrm{~nm}, 77 \mathrm{~nm}$, and $83 \mathrm{~nm}$. The tuning ranges of the setup using the CFBG are narrower than that using the DCF. This is because the reflection bandwidth (FWHM) of the CFBG is $100 \mathrm{~nm}$, and the tuning range of the laser is limited to less than $100 \mathrm{~nm}$. The tuning range around $80 \mathrm{~nm}$ corresponds to the axial resolution of $13 \mu \mathrm{m}$ using (13). Since the dispersion-tuned laser does not have the Gaussian spectral envelope, the actual resolution would be lower. Figures 11(d) - 11(f) show the relation between the lasing wavelength and the mode-locking frequency. We found that the lasing wavelength is linearly proportional to the mode-locking frequency.
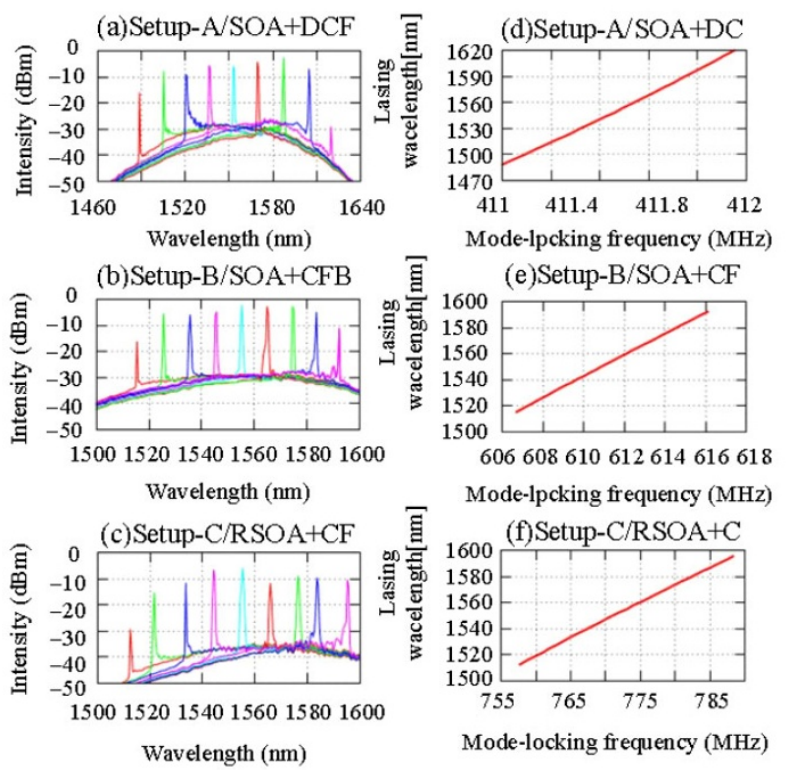

Fig. 11 Static characteristics: (a)-(c) the lasing spectra of the setup-A, setup-B, and setup-C, and (d)-(f) the relation between the lasing wavelength and the mode-locking frequency in the setup-A, setup-B, and setup-C.

In the dispersion tuning method, the change in the optical frequency, rather than the wavelength, is proportional to the mode-locking frequency when the higher-order dispersions $\left(\beta_{3}, \beta_{4}, \ldots\right)$ are negligible, as shown in (8). Thus, the dispersion- tuned laser having only 1st-order dispersion is suitable for the SS-OCT system, which requires the linear frequency sweep for the linear sampling in the optical frequency domain. The average static linewidths of the setup-A, setup-B, and setup-C are $0.59 \mathrm{~nm}, 0.45 \mathrm{~nm}$, and $0.32 \mathrm{~nm}$, respectively. The linewidth becomes narrower as the cavity length become shorter. We presume this is due to the use of the higher mode-locking frequency for the shorter cavity.

Figure 12 shows the sweep characteristics obtained by inputting the saw-tooth wave from the FG and sweeping the mode-locking frequency linearly. Figures 12(a) - 12(c) show the peak-hold spectra at the sweep rate of $1 \mathrm{kHz}, 10 \mathrm{kHz}, 100 \mathrm{kHz}$, and $1 \mathrm{MHz}$. A decrease in the optical spectra at high sweep rates is because of the integral time of the peak-hold function of the OSA. The ripples observed in Figs. 12(a) - 12(c) are presumably due to the modulation characteristics of the SOA and RSOA. The sweep range of the setup-A is intentionally reduced to $80 \mathrm{~nm}$ in order to compare the sweep characteristics with the other two setups under the same spatial resolution. The peak-hold spectrum at $1 \mathrm{MHz}$ of the setup-A collapses due to its long cavity length. The sweep range of the setup-C shown in Fig. $12(\mathrm{c})$ is about $60 \mathrm{~nm}$, which is narrower than the tuning range shown in Fig. 11(c). We had to limit the sweep range to $60 \mathrm{~nm}$. The sweep of the modulation frequency $\Delta f_{m}$ needed for a specific sweep range is inversely proportional to the cavity length as indicated in (2), and our RF synthesizer and FG are not capable of covering the entire 83-nm range. The output powers of the setup-A, setup-B, and setup-C are $9.5 \mathrm{~mW}, 4.9 \mathrm{~mW}$, and $8.4 \mathrm{~mW}$, respectively.

Figures 12(d) - 12(f) show the temporal waveforms at $1 \mathrm{kHz}, 10 \mathrm{kHz}, 100 \mathrm{kHz}$, and $1 \mathrm{MHz}$. The voltage of the setup-A output shown in Fig. 12(d) decreases as the sweep rate becomes faster, and no signal was observed at the sweep rate of $1 \mathrm{MHz}$. The waveforms of the setup-B shown in Fig. 
12(e) is superior to that of the setup-A. However, the signal could hardly be observed at $1 \mathrm{MHz}$. The sweep waveforms of the setup-C are much better than the others. As shown in Fig. 12(f), the output power of the setup-C remains high at the fast sweep speed of $1 \mathrm{MHz}$. This indicates that this laser can work at the high sweep rate exceeding $100 \mathrm{kHz}$. The performances of each setup are summarized in Table 1. The static linewidth and the laser performance at high sweep rates become better as the laser becomes shorter.

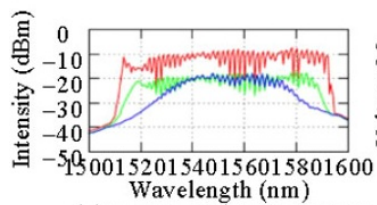

(a) Setup-A/SOA+DCF

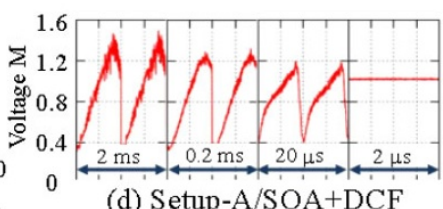

(d) Setup-A/SOA+DCF
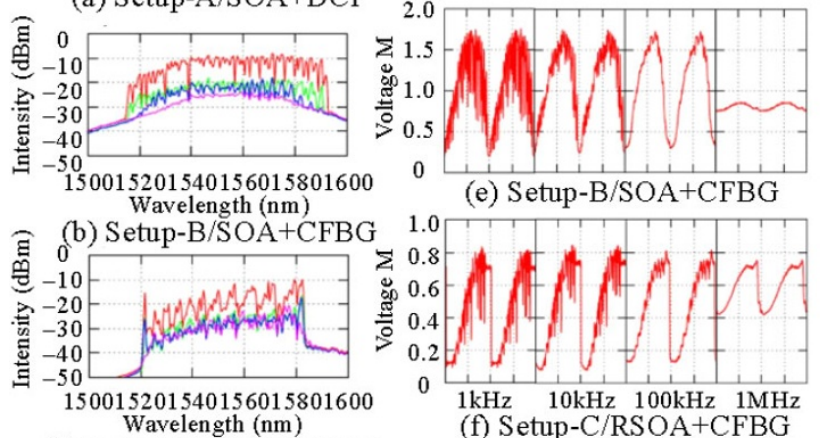

(c) Setup-C/RSOA+CFBG

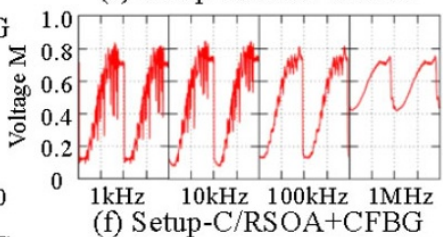

Fig. 12 Dynamic characteristics: (a)-(c) peak-hold spectra of the setup-A, setup-B, and setup-C (from top to bottom: $1 \mathrm{kHz}$, $10 \mathrm{kHz}, 100 \mathrm{kHz}$, and $1 \mathrm{MHz}$ ), and (d)-(f) temporal waveform of setup-A, setup-B, and setup-C.

Table 1 Summary of performances of three lasers.

\begin{tabular}{c|c|c|c}
\hline & $\begin{array}{c}\text { Setup-A } \\
\text { SOA+DCF }\end{array}$ & $\begin{array}{c}\text { Setup-B } \\
\text { SOA+CFBG }\end{array}$ & $\begin{array}{c}\text { Setup-C } \\
\text { RSOA+CFBG }\end{array}$ \\
\hline Cavity length & $105.2 \mathrm{~m}$ & $9.8 \mathrm{~m}$ & $4.1 \mathrm{~m}$ \\
\hline Tuning range & $131 \mathrm{~nm}$ & $77 \mathrm{~nm}$ & $83 \mathrm{~nm}$ \\
\hline Static linewidth & $0.59 \mathrm{~nm}$ & $0.45 \mathrm{~nm}$ & $0.32 \mathrm{~nm}$ \\
\hline $\begin{array}{c}\text { Sweep range } \\
\text { (at 1-kHz sweep) }\end{array}$ & $80 \mathrm{~nm}$ & $77 \mathrm{~nm}$ & $60 \mathrm{~nm}$ \\
\hline $\begin{array}{c}\text { Output power } \\
\text { (at 1-kHz sweep) }\end{array}$ & $9.5 \mathrm{~mW}$ & $4.9 \mathrm{~mW}$ & $8.4 \mathrm{~mW}$ \\
\hline High sweep rate & Poor & Moderate & Good \\
\hline
\end{tabular}

\subsection{Application to SS-OCT}

We applied the dispersion-tuned wavelength- swept fiber laser to the OCT system shown in Fig. 7. Figure 13 shows the point spread function (PSF) of the laser at the sweep rate of $1 \mathrm{kHz}$ and $50 \mathrm{kHz}$. The PSF is measured with a Michelson interferometer in Fig. 7 by placing and moving a $90 \%$ mirror at the probe arm. The amplitude of the interference fringes decreases as the optical path difference is longer according to the coherence length, which is equal to the imaging depth. Figures 13(a)-13(c) show the PSF of the setup-A, setup-B, and setup-C at the $1-\mathrm{kHz}$ sweep rate. The axial resolution of the systems read from the FWHM of the signal in PSF is about $30 \mu \mathrm{m}$, which is nearly the same for all setups. As the theoretical value calculated from (4) is about $10 \mu \mathrm{m}$, the axial resolution of the OCT system is three times larger than the theoretical value. We attribute it to the mismatch between the laser and the adopted OCT system, i.e., the laser is pulsed whereas the OCT system is optimized for continuous-wave laser operation. Figures 13(a)-13(c) indicate that the depth range of the setup-A is worse than the other two setups. The coherence lengths of each system calculated from (5) are $1.7 \mathrm{~mm}, 2.2 \mathrm{~mm}$, and $3.1 \mathrm{~mm}$, and those measured from the PSF are $0.6 \mathrm{~mm}, 2.2 \mathrm{~mm}$, and 1.6 $\mathrm{mm}$. The setup using the normal SOA and the CFBG shows the best drop-off characteristics. The measured coherence lengths of other two setups are worse than the calculated value, which indicates the linewidth broadening in the wavelength sweep. In the setup-A, it is because of a long cavity length. In the setup-C, the linewidth is probably not constant through the sweep due to the modulation efficiency of the RSOA.

Figures 13(d) - 13(f) show the PSF of the setup-A, setup-B, and setup-C at the $50-\mathrm{kHz}$ sweep rate. As shown in Fig. 13(d), the signal drop-off of the setup-A is not good, and we could observe the signal only near the surface. The performance of the setup-B shown in Fig. 13(e) is better than that of the setup-A. Still, the coherence length of the setup-B is 
about $0.5 \mathrm{~mm}$, which is worse than that of the $1-\mathrm{kHz}$ sweep. By contrast, the drop-off characteristics of the setup-C are better, as indicated in Fig. 13(f). The coherence length is about $0.8 \mathrm{~mm}$, which is better than that of the other two setups.
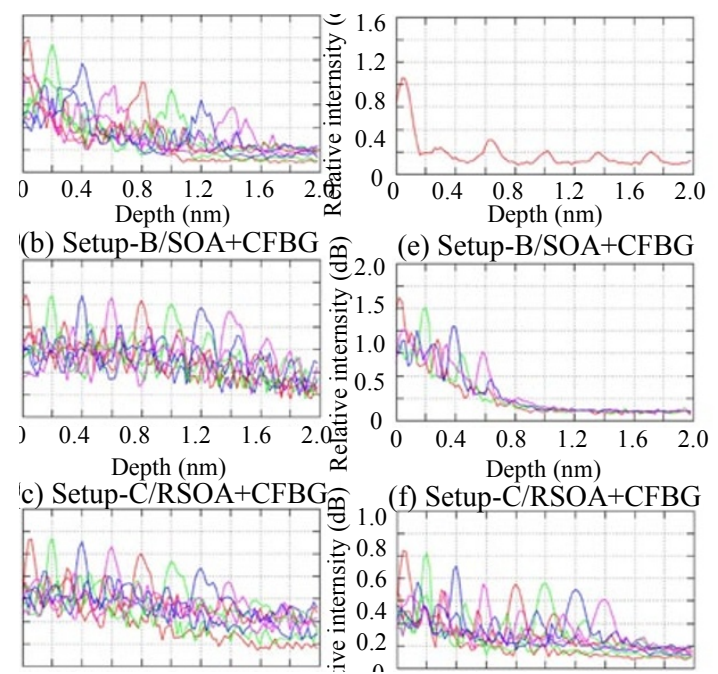

Fig. 13 Point spread function (a)-(c) at $1 \mathrm{kHz}$ [(a) setup-A, (b) setup-B, and (c) setup-C] and (d)-(f) at $50 \mathrm{kHz}$ [(d) setup-A, (e) setup-B, and (f) setup-C].

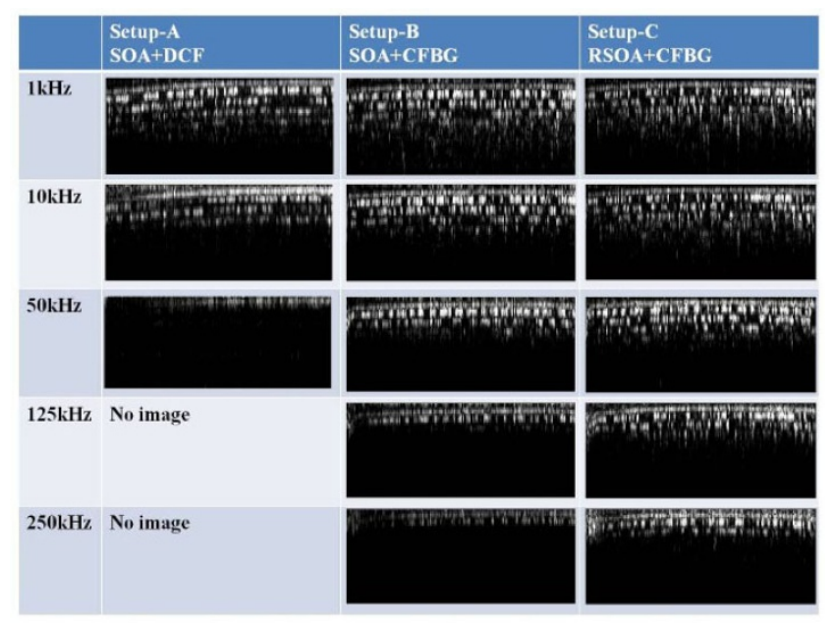

Fig. 14 OCT images of an adhesive tape $(1 \mathrm{~mm} \times 10 \mathrm{~mm})$.

Figure 14 shows the OCT images of an adhesive tape obtained by the system. In each image, several layers from the surface were observed. As shown in Fig. 14, we could obtain the image at the sweep rate of up to $10 \mathrm{kHz}$ using the setup-A. At $50 \mathrm{kHz}$, however, we could observe only the surface and could not penetrate inside the tape. We could obtain the image at up to the $250-\mathrm{kHz}$ sweep rate using the setup-B, although the images obtained at $125 \mathrm{kHz}$ and $250 \mathrm{kHz}$ were poor. In contrast, images from the setup-C remained relatively good even at high sweep rates. The short cavity enhanced the stability at the high sweep rate.

\section{Conclusions}

In this review paper, we described the principle of the dispersion-tuned swept lasers in detail and presented our recent work on the application to the SS-OCT system. We demonstrated that the dispersion-tuned swept lasers are applicable to the SS-OCT system and also that the performance at high sweep rates can be significantly enhanced by making the cavity length short with the RSOA and CFBG. We successfully obtained OCT images at up to the $250-\mathrm{kHz}$ sweep. A remaining issue for the SS-OCT application is the short coherence length of the laser, especially at higher sweep rates, due to the broadening of the instantaneous linewidth of the laser. One solution is the use of a dispersive element with anomalous dispersion [27]. The combination of anomalous dispersion and self-phase modulation in the SOA has been demonstrated to be able to narrow the linewidth of the laser [23-25, 27]. We are working on improving the coherence length of the laser at higher sweep rates.

\section{Acknowledgment}

The authors wish to thank Mr. Masataka Tei of Santec Corp. for cooperation on the OCT experiment. This work was supported by the Funding Program for Next Generation WorldLeading Researchers (NEXT Program) of the Japan Society for the Promotion of Science (JSPS).

Open Access This article is distributed under the terms of the Creative Commons Attribution License which permits any use, distribution, and reproduction in any 
medium, provided the original author(s) and source are credited.

\section{References}

[1] D. Huang, E. A. Swanson, C. P. Lin, J. S. Schuman, W. G. Stinson, W. Chang, et al., "Optical coherence tomography," Science, vol. 254, no. 5035, pp. 1178-1181, 1991.

[2] S. R. Chinn, E. A. Swanson, and J. G. Fujimoto, "Optical coherence tomography using a frequencytunable optical source," Optics Letters, vol. 22, no. 5, pp. 340-342, 1997.

[3] B. Potsaid, B. Baumann, D. Huang, S. Barry, A. E. Cable, J. S. Schuman, et al., "Ultrahigh speed $1050 \mathrm{~nm}$ swept source / Fourier domain OCT retinal and anterior segment imaging at 100000 to 400000 axial scans per second," Optics Express, vol. 18, no. 19, pp. 20029-20048, 2010.

[4] W. Y. Oh, S. H. Yun, G. J. Tearney, and B. E. Bouma, "115 kHz tuning repetition rate ultrahighspeed wavelength-swept semiconductor laser," Optics Letters, vol. 30, no. 23, pp. 3159-3161, 2005.

[5] W. Y. Oh, B. J. Vakoc, M. Shishkov, G. J. Tearney, and B. E. Bouma, " $>400 \mathrm{kHz}$ repetition rate wavelength-swept laser and application to high-speed optical frequency domain imaging," Optics Letters, vol. 35 , no. 17 , pp. 2919-2921, 2010 .

[6] R. Huber, M. Wojtkowski, K. Taira, and G. Fujimoto, "Amplified, frequency swept lasers for frequency domain reflectometry and OCT imaging: design and scaling principles," Optics Express, vol. 13, no. 9, pp. 3513-3528, 2005.

[7] M. Kuznetsov, W. Atia, B. Johnson, and D. Flanders, "Compact ultrafast reflective Fabry-Perot tunable lasers for OCT imaging applications," in Proc. SPIE, vol. 7554, pp. 75541F-1-75541F-6, 2010.

[8] R. Huber, M. Wojtkowski, and J. G. Fujimoto, "Fourier domain mode locking (FDML): a new laser operating regime and applications for optical coherence tomography," Optics Express, vol. 14, no. 8, pp. 3225-3237, 2006.

[9] M. Y. Jeon, J. Zhang, Q. Wang, and Z. Chen, "High-speed and wide bandwidth Fourier domain mode-locked wavelength swept laser with multiple SOAs," Optics Express, vol. 16, no. 4, pp. 2547-2554, 2008.

[10] S. Marschall, T. Klein, W. Wieser, B. R. Biedermann, K. Hsu, K. P. Hansen, et al., "Fourier domain mode-locked swept source at $1050 \mathrm{~nm}$ based on a tapered amplifier," Optics Express, vol. 18, no. 15, pp. 15820-15831, 2010.

[11] W. Wieser, B. R. Biedermann, T. Klein, C. M. Eigenwillig, and R. Huber, "Multi-megahertz OCT: high quality $3 \mathrm{D}$ imaging at 20 million A-scans and 4.5 Gvoxels per second," Optics Express, vol. 18, no. 14, pp. 14685-14704, 2010.

[12] V. Jayaraman, J. Jiang, H. Li, P. J. S. Heim, G. D. Cole, B. Potsaid, et al., "OCT Imaging up to $760 \mathrm{kHz}$ axial scan rate using single-mode $1310 \mathrm{~nm}$ MEMS-tunable VCSELs with $>100 \mathrm{~nm}$ tuning range," presented at 2011 Conference on Lasers and Electro-Optics, Baltimore, USA, May 1-6, 2011.

[13] S. Yamashita and M. Asano, "Wide and fast wavelength-tunable mode-locked fiber laser based on dispersion tuning," Optics Express, vol. 14, no. 20, pp. 9299-9306, 2006.

[14] Y. Nakazaki and S. Yamashita, "Fast and wide tuning range wavelength-swept fiber laser based on dispersion tuning and its application to dynamic FBG sensing," Optics Express, vol. 17, no. 10, pp. 8310-8318, 2009.

[15] S. Yamashita, Y. Nakazaki, R. Konishi, and O. Kusakari, "Wide and fast wavelength-swept fiber laser based on dispersion tuning for dynamic sensing (invited)," Journal of Sensors, A Special Issue on Fiber and Integrated WaveguideBased Optical Sensors, vol. 2009, pp. 572835, 2009.

[16] Y. Takubo and S. Yamashita, "In vivo OCT imaging using wavelength-swept fiber laser based on dispersion tuning," IEEE Photonics Technology Letters, vol. 24, no. 12, pp. 979-981, 2012.

[17] Y. Takubo and S. Yamashita, "High-speed dispersion-tuned wavelength-swept fiber laser using a reflective SOA and a chirped FBG," Optics Express, vol. 21, no. 4, pp. 5130-5139, 2013.

[18] G. P. Agrawal, Applications of nonlinear fiber optics. New York: Academic Press, 2001.

[19] S. Li and K. T. Chan, "Electrical wavelength tunable and multiwavelength actively mode-locked fiber ring laser," Applied Physics Letters., vol. 72, no. 16, pp. 1954-1956, 1998.

[20] K. Tamura and M. Nakazawa, "Dispersion-tuned harmonically mode-locked fiber ring laser for self-synchronization to an external clock," Optics Letters, vol. 21, no. 24, pp.1984-1986, 1996.

[21] F. Lexer, C. K. Hitzenberger, A. F. Fercher, and M. 
Kulhavy, "Wavelength-tuning interferometry of intraocular distances," Applied Optics, vol. 36, no. 25, pp. 6548-6553, 1997.

[22] C. Chong, T. Suzuki, A. Morosawa, and T. Sakai, "Spectral narrowing effect by quasi-phase continuous tuning in high-speed wavelength-swept light source," Optics Express, vol. 16, no. 25, pp. 21105-21118, 2008.

[23] B. Burgoyne and A. Villeneuve, "Programmable lasers: design and applications," in Proc. SPIE, vol. 7580, pp. 758002-1-758002-15, 2010.

[24] G. Lamouche, S. Vergnole, Y. Kim, B. Burgoyne, and A. Villeneuve, "Tailoring wavelength sweep for SS-OCT with a programmable picosecond laser," in Proc. SPIE, vol. 7889, pp. 78891L-1-78891L-6,
2011.

[25] Y. Kim, B. Burgoyne, N. Godbout, A. Villeneuve, G. Lamouche, and S. Vergnole, "Picosecond programmable laser sweeping over 50 megawavelengths per second," in Proc. SPIE, vol. 7914, pp. 79140Y-1-79140Y-8, 2011.

[26] H. D. Lee, J. H. Lee, M. Y. Jeong, and C. S. Kim, "Characterization of wavelength-swept active mode locking fiber laser based on reflective semiconductor optical amplifier," Optics Express, vol. 19, no. 15, pp. 14586-14593, 2011.

[27] A. Takada, M. Fujino, and S. Nagano, "Dispersion dependence of linewidth in actively mode-locked ring lasers," Optics Express, vol. 20, no. 4, pp. 4753-4762, 2012. 